\title{
TITLE:
}

\section{Double-resonance magic angle coil spinning}

AUTHOR(S):

Inukai, Munehiro; Takeda, Kazuyuki

CITATION:

Inukai, Munehiro ...[et al]. Double-resonance magic angle coil spinning. Journal of Magnetic Resonance 2010, 202 (2): 274-278

ISSUE DATE:

2010-02

URL:

http://hdl.handle.net/2433/91254

RIGHT:

c 2009 Elsevier Inc. All rights reserved. 


\title{
Double-resonance magic angle coil spinning
}

\author{
Munehiro Inukai ${ }^{a}$, Kazuyuki Takeda ${ }^{\mathrm{a}, *}$, \\ ${ }^{a}$ Division of Chemistry, Graduate School of Science, Kyoto University, 606-8502 \\ Kyoto, Japan
}

\begin{abstract}
We present an extension of magic angle coil spinning (MACS) solid-state NMR spectroscopy to double-resonance experiments, enabling implementation of powerful double-resonance solid-state NMR methodologies including cross polarization, proton decoupling, and two-dimensional correlation spectroscopy etc., while still enjoying the merits that are intrinsic to MACS, such as high concentration sensitivity, eliminated magnetic susceptibility-induced field distortion, and an easy-to-use approach with the conventional and widespread hardware.
\end{abstract}

Key words: microcoil, magic angle spinning (MAS), magic angle coil spinning (MACS), double-resonance

Studies of molecular structure and motion by means of NMR spectroscopy are powerful but the sensitivity is low. In many applications of NMR to materials of chemical/biological interest, the concentration sensitivity, i.e., the sensitivity per unit sample volume, is often more important than that of the mass sensitivity, and the need to gain the former in NMR analysis of sparse, volume-limited samples stimulated development of a tiny detection coil called * corresponding author Email address: takezo@kuchem.kyoto-u.ac.jp (Kazuyuki Takeda). 
as a microcoil, which was firstly applied to investigate liquid samples with a volume of as small as 5 nanoliters[1], and then to stationary solid materials with less than a milligram[2,3]. By reducing the size of the coil one can significantly enhance the filling factor (the ratio of the sample volume to the coil volume) and thereby the attainable concentration sensitivity $[4,5]$.

The standard and widespread approach for high-resolution NMR of polycrystalline and amorphous materials demands Magic Angle Spinning (MAS)[6,7], which has recently stimulated its combination with microcoil NMR in the context of concentration sensitivity. Among several reports on microcoil-MAS NMR spectroscopy[8-10], a remarkable idea put forth by Sakellariou et al. involves spinning of the microcoil together with the sample of interest, and wireless, inductive signal transmission between the spinning microcoil and and the primary circuit of the MAS probe[11]. In this outstanding strategy, referred to as Magic Angle Coil Spinning (MACS), the coil can be wound directly around the capillary sample so as to gain the filling factor, while inhomogeneous magnetic field distortion due to bulk magnetic susceptibility of the wire of the microcoil is eliminated by spinning the microcoil at the magic angle. Furthermore, the importance of the MACS approach cannot be overstated from the practical point of view, because it requires no other than a standard, popularly used MAS probe, while any other approaches for microcoil MAS[8-10] do require some hardware modification inside the MAS probe. Thus, the idea of MACS provides a versatile strategy toward high-resolution solid-state NMR measurements with enhanced concentration sensitivity for mass-limited samples.

Currently, the MACS experiments are demonstrated in ${ }^{1} \mathrm{H}$ MAS NMR, ${ }^{29} \mathrm{Si}$ NMR, and ${ }^{23} \mathrm{Na}$ multiple-quantum MAS NMR[11], while it is often required 
in solid-state NMR spectroscopy to simultaneously handle two nuclear spin species having different gyromagnetic ratios and thereby different resonance frequencies. For example, in many solid-state ${ }^{13} \mathrm{C}$ NMR studies of biomolecules and polymers, the ${ }^{13} \mathrm{C}$ magnetization is enhanced by means of Cross Polarization (CP)[12]. Also, the ${ }^{13} \mathrm{C}$ magnetization is usually observed after $\mathrm{CP}$ under both MAS and intense rf irradiation at the ${ }^{1} \mathrm{H}$ spin for the purpose of decoupling, i.e., of eliminating the dipolar interaction between the ${ }^{13} \mathrm{C}$ and the ${ }^{1} \mathrm{H}$ spins so as to enhance spectral resolution. In this work, we integrate MACS and double-resonance NMR spectroscopy into an easy-to-use, powerful, and sensitive analytical tool for intrinsically mass-limited polycrystalline and amorphous solid materials with a small volume of the order of $0.1 \mathrm{~mm}^{3}$ or less, opening a new arena of chemical analysis in which one can enjoy benefits from both MACS and double-resonance NMR spectroscopy. Here we demonstrate ${ }^{1} \mathrm{H}-{ }^{13} \mathrm{C}$ double-resonance MACS experiments in a magnetic field of $7 \mathrm{~T}$. All NMR spectra presented in this work were acquired using an OPENCORE NMR spectrometer[13], which is a home-built, FPGA-based NMR spectrometer[14].

Fig. 1a describes a circuit diagram for a doubly-tuned MACS resonator, to be put inside a rotor and spun together with the rotor in the conventional doubly-tuned MAS probe. The doubly-tuned MACS resonator is composed of two capacitors $C_{1}$ and $C_{2}$ and two inductors $L_{1}$ and $L_{2}$, and their mutual inductance is denoted by $M . V_{1}$ and $V_{2}$ represent electromotive forces induced by the primary circuit (not described in the figure) in the probe through the inductive couplings. Using the first and second Kirchhoff's laws, we obtain an equation of resonance for a given set of $L_{1}, L_{2}, M, C_{1}, C_{2}$, and the resonance 
frequency $\omega$ :

$$
0=1-\left[L_{1} C_{1}+\left(L_{1}+L_{2}+2 M\right) C_{2}\right] \omega^{2}+C_{1} C_{2}\left(L_{1} L_{2}-M^{2}\right) \omega^{4} .
$$

By setting the individual resonance frequencies to the Larmor frequencies $\omega_{I}$ and $\omega_{S}$ of the nuclear spin species under interest, double-resonance NMR is realized.

We wound two coils (inner diameter: $0.5 \mathrm{~mm}$ ) with $80 \mu \mathrm{m}$ polyurethane-coated copper wire, and assembled the doubly-tuned MACS resonator in an axially symmetric form using two chip capacitors, as shown in Fig. 1b. As discussed extensively by the Sakellariou group in their pioneering papers[11,15], the eddy current induced in the spinning coils and capacitors can have a considerable sample-heating effect. In order to minimize the amount of metal and thus creation of heat, we employed spot welding as a means of contacting the wire onto the electrodes of the chip capacitor, since it does not add any extra metal unlike soldering. Also, for effective heat dissipation, Shapal-M, machinable ceramic known to have an excellent thermal conductivity, was used[11] for the spacer holding the MACS resonator inside a spinning sample tube.

Fig. 1(c) illustrates the Shapal-M insert for a Varian $5 \mathrm{~mm}$ rotor. It is composed of two parts sandwiching the two chip capacitors, which were placed in a way that they balance each other. Using a Varian $5 \mathrm{~mm}$ triple-resonance CP-MAS probe, we verified stable spinning at up to $10 \mathrm{kHz}$ both inside and outside a wide-bore $7 \mathrm{~T}$ superconducting magnet. We cautiously did not try to spin it at the certificated maximum spinning speed of $12 \mathrm{kHz}$.

We put the two coils apart from each other, so that their coupling $M$ is negligible. We then obtain from Eq. (1) 


$$
\begin{aligned}
& L_{1}=\frac{\beta C_{1} \pm \sqrt{\beta^{2} C_{1}^{2}-4 \alpha C_{1}\left(C_{1}+C_{2}\right)}}{2 C_{1}\left(C_{1}+C_{2}\right)}, \\
& L_{2}=\frac{\beta C_{1} \mp \sqrt{\beta^{2} C_{1}^{2}-4 \alpha C_{1}\left(C_{1}+C_{2}\right)}}{2 C_{1} C_{2}},
\end{aligned}
$$

where

$$
\begin{aligned}
& \alpha=\left(\omega_{I} \omega_{S}\right)^{-2}, \\
& \beta=\omega_{I}^{-2}+\omega_{S}^{-2} .
\end{aligned}
$$

In this work we deal with ${ }^{1} \mathrm{H}-{ }^{13} \mathrm{C}$ double-resonance NMR experiments in a magnetic field of $7 \mathrm{~T}$, and in Fig. 2a-b, such $L_{1}$ and $L_{2}\left(L_{1}<L_{2}\right)$ are plotted for various capacitances $C_{1}$ and $C_{2}$ that the circuit resonates at $300 \mathrm{MHz}$ and $75 \mathrm{MHz}$, which correspond to the ${ }^{1} \mathrm{H}$ and ${ }^{13} \mathrm{C}$ Larmor frequencies, respectively. As seen in Fig. 2a-b, it is possible to attain double-resonance by adjusting $L_{1}$ and $L_{2}$ for a wide range of the capacitances $C_{1}$ and $C_{2}$, so that capacitance tolerance can be compensated. We used $L_{2}$ as the sample coil, into which we inserted a capillary sample tube (outer diameter: $0.5 \mathrm{~mm}$, inner diameter: 0.3 $\mathrm{mm}$ ) suitable for samples with a volume of $\sim 0.1 \mathrm{~mm}^{3}$.

In the present work we have chosen the values of $C_{1}, C_{2}, L_{1}$, and $L_{2}$ as follows. Firstly we tentatively set the length of the sample coil $L_{2}$ to ca. $2.5 \mathrm{~mm}$, which, with its inner diameter determined by the capillary outer diameter of $0.5 \mathrm{~mm}$ and a number of turns of ca. 18, gives its inductance to be $\sim 40 \mathrm{nH}$. Then, according to the region close to the $40 \mathrm{nH}$ contour line in Fig. 2b, we have chosen the capacitances out of the standard capacitances values to be $C_{1}=33$ $\mathrm{pF}$ and $C_{2}=82 \mathrm{pF}$, from which $L_{1}=11 \mathrm{nH}$ and $L_{2}=43 \mathrm{nH}$ were obtained from Eqs. (2)-(3).

After fabricating the resonator, we slightly shrinked and stretched the coils 
several times until it resonated at the desired frequencies of 300 and $75 \mathrm{MHz}$. The resonance frequencies were checked by putting the MACS insert into the primary coil of the CP-MAS probe that was in advance tuned and matched using a network analyzer. When the resonance frequencies of the insert was close to that of the primary circuit, considerable deviation of the reflection coefficient was observed, while deviation as increasing the difference in the resonance frequencies. After verifying the resonance frequencies of the MACS insert, the final impedance matching was attained by adjusting the trimmer capacitors of the primary circuit so that the reflection coefficients at the relevant frequencies were minimized.

Frequency mismatch of the MACS insert can apparently be compensated by adjusting the trimmer capacitors of the primary probe so as to give the minimum reflection coefficient. However, it should be small for efficient inductive rf transmission at the Larmor frequency. In order to study the mismatch tolerance, we examined the efficiencies of nutation for various resonance frequencies of the MACS insert over a range of $10 \mathrm{MHz}$ around the ${ }^{13} \mathrm{C}$ resonance frequency of $\sim 75 \mathrm{MHz}$. We then found that mismatch by less than $\sim \pm 1 \mathrm{MHz}$ did not significantly disturb the performance. On the other hand, the rf efficiency degraded considerably for larger mismatch. Thus, we made a rough estimation of the $\mathrm{Q}$ factor of the present MACS resonator at the ${ }^{13} \mathrm{C}$ channel to be $\sim 40$. It would be worth mentioning here that the width of this mismatch tolerance does not coincide with the frequency bandwidth of $-3 \mathrm{~dB}$ return loss measured for the overall circuit, and we have not yet found a straightforward way of accurately measuring the Q factor of the doubly-tuned MACS resonator alone that gives us the mismatch tolerance bandwidth.

In order to evaluate sensitivity enhancement introduced by employing the 
doubly-tuned MACS resonator, we examined the frequencies of nutation for both the ${ }^{1} \mathrm{H}$ and ${ }^{13} \mathrm{C}$ channels of the Varian $5 \mathrm{~mm}$ CP-MAS probe with and without the tuned double-resonance MACS insert shown in Fig. 1. According to the principle of reciprocity[4], the electromotive force induced in the sample coil by a magnetic dipole at a point is proportional to the magnetic field created by a unit current in the coil at the same point. It follows that dependence of the nutation frequency on the power of $\mathrm{rf}$ irradiation gives a direct measure of the signal intensity for a given nuclear magnetization. In Fig. 3a, the ${ }^{1} \mathrm{H}$ and ${ }^{13} \mathrm{C}$ nutation frequencies are plotted for various radiofrequency powers, showing the enhancement factors of $5.4(=46.2 / 8.60)$ and $7.0(=26.6 / 3.80)$ for the ${ }^{1} \mathrm{H}$ and ${ }^{13} \mathrm{C}$ channels. Fig. $3 \mathbf{b}$ and $\mathbf{c}$ show ${ }^{13} \mathrm{C}$ CP-MAS spectra of an identical polycrystalline sample of uniformly ${ }^{13} \mathrm{C}-{ }^{15} \mathrm{~N}$-labeled L-alanine, where the sample was put into the rotor as usual in $\mathbf{b}$, while the doubly-tuned MACS resonator was filled with the sample in c. As expected, the latter resulted in an enhancement factor of ca. 7 in the ${ }^{13} \mathrm{C}$ NMR signal intensity, in good agreement with the enhancement factor in the ${ }^{13} \mathrm{C}$ nutation efficiency described in Fig. 3a. Note, however, that the microcoil resistance causes additional noise, so that the overall enhancement factor in the signal-to-noise ratio was less than the enhancement factor in the signal intensity $(6.3,6.6$, and 6.3 in the present case for the methyl, methine, and carboxyl carbons, respectively).

In Fig. 3b, a weak background signal is visible at around $110 \mathrm{ppm}$. Since this peak does not appear in the MACS spectrum of Fig. 3c, it should come from the ${ }^{13} \mathrm{C}$ spins outside the coils of the MACS insert. From the nutation experiment we also estimated the rf-field distribution experienced by the sample inside the MACS resonator ranges over ca. $11 \%$.

Since the signal-to-noise ratio is proportional to the square root of the num- 
ber of signal accumulations, this result indicates that double-resonance MACS accelerates the experimental time by a factor of 41 compared to the same measurement with the conventional approach, making double-resonance solid-state MAS NMR of sparse materials feasible, and importantly, it has been possible simply by putting the doubly-tuned MACS resonator into a commercially available, standard and widespread double-resonance MAS probe without any hardware modification. Fig. $4 \mathbf{a}$ shows a ${ }^{13} \mathrm{C}$ CP-MAS spectrum of partially ${ }^{13} \mathrm{C}-{ }^{15}$ N-labeled 42 -mer amyloid $\beta$ peptide $(\mathrm{A} \beta 42)[16]$, known to play a critical role in the etiology of Alzheimer's disease. Here the sample amount was $\sim 0.1$ mg, and the spectrum was obtained with double-resonance MACS by accumulating the ${ }^{13} \mathrm{C}$ NMR signal for 2.08 days, while the expected experimental time required to attain the same signal-to-noise ratio with the conventional probe exceeds 85 days.

Fig. 4b shows another example of double-resonance MACS demonstrating two-dimensional ${ }^{13} \mathrm{C}-{ }^{13} \mathrm{C}$ correlation experiment by means of dipolar-assisted rotational resonance (DARR) $[17,18]$ in polycrystalline ${ }^{13} \mathrm{C}$-labeled histidine. Multi-dimensional NMR is, even though powerful for extracting secondary structure of biomolymers, time consuming, so that a considerable amount of sample is usually required to reduce the number of signal accumulations. In many applications, however, samples with ${ }^{13} \mathrm{C}$ labelling at specific sites have to be prepared to assign the resonance lines and obtain distance restrains, and synthesis of a number of differently labeled samples with quantities giving tolerable sensitivity in the conventional solid-state NMR is often formidable. Double-resonance MACS presented here is also promising in this context, since it allows us to obtain two-dimensional correlation spectra with much less sample amount, as demonstrated in Fig. $\mathbf{4 b}$. 
For a static microcoil, the susceptibility effect of the coil material causes static field distortion in the coil proximity, and it was demonstrated that spinning of the MACS insert effectively eliminated resonance-line broadening induced by the susceptibility of the microcoil as well as that of the biological tissue sample[11]. In order to examine the effect of coil susceptibility in ${ }^{1} \mathrm{H}_{-}{ }^{13} \mathrm{C}$ doubleresonance MACS in comparison with the case of a static microcoil, we carried out another microcoil-MAS experiment with the piggy-back scheme[8] using the same $7 \mathrm{~T}$ superconducting magnet and under the same experimental condition, i.e., the identical polycrystalline sample of ${ }^{13} \mathrm{C}-{ }^{15} \mathrm{~N}$-labeled L-alanine, the same pulse sequence and rf intensities, and the same spinning speed. The diameter of the static microcoil was $0.8 \mathrm{~mm}$, and the outer and inner diameters of the sample capillary were 0.4 and $0.3 \mathrm{~mm}$. Our piggy-back microcoil-MAS probe is described in Ref. [10] in detail. As demonstrated in Fig. 5 showing the methyl ${ }^{13} \mathrm{C}$ peaks, ${ }^{13} \mathrm{C}-{ }^{13} \mathrm{C} \mathrm{J}$ splitting could be obserbed in MACS, while broadening caused by the coil susceptibility obscured the structure in the piggy-back scheme, indicating that susceptibility-matching[1] would be required in the latter.

The MACS resonator, whether singly- or doubly-tuned, is transparent to radiofrequency fields for a wide range of frequencies outside the resonance bands. It is thus possible to use an extra channel of the primary circuit in the probe to put another nuclear spin species in the experiment. With a double-resonance MAS NMR probe, a singly tuned MACS resonator for one nuclear spin species can be inductively coupled with one of the two channels in the probe, while the other channel can also be used for another species. Similarly, with a tripleresonance probe, we would be able to perform double-resonance MACS with two of the three channels, and use the other for another, third nuclear spin 
species. Even though the filling factor for the third channel is poor, not all nuclear spin species are subject to observation in most triple-resonance NMR experiments, so that the additional channel may be assigned to the unobserved spin species. For example, in ${ }^{1} \mathrm{H}_{-}{ }^{13} \mathrm{C}-{ }^{15} \mathrm{~N}$ triple-resonance sequences used frequently for the study of ${ }^{13} \mathrm{C}-{ }^{15} \mathrm{~N}$-labeled peptides and proteins, the ${ }^{1} \mathrm{H}$ spins are only irradiated for the purpose of magnetization transfer and decoupling, while it is essential to detect the ${ }^{13} \mathrm{C}$ and ${ }^{15} \mathrm{~N}$ NMR signals with high concentration sensitivity. On the other hand, for strong ${ }^{1} \mathrm{H}$ decoupling which is only possible with the microcoil[8], it would be necessary to extend MACS to triple-resonance by fabricating a triply-tuned resonator based on the circuit diagram depicted in Fig. 6, which involves three inductors $L_{1}, L_{2}$, and $L_{3}$ and three capacitors $C_{1}, C_{2}$, and $C_{3}$. For the case of negligible mutual inductances among the inductors, the resonance condition is given by

$$
\begin{aligned}
0= & 1-\left[L_{1} C_{1}+\left(L_{1}+L_{2}\right) C_{2}+\left(L_{1}+L_{2}+L_{3}\right) C_{3}\right] \omega^{2} \\
& +\left[L_{1} L_{2} C_{1} C_{2}+\left(L_{1}+L_{2}\right) L_{3} C_{2} C_{3}+L_{1}\left(L_{2}+L_{3}\right) C_{1} C_{3}\right] \omega^{4} \\
& -L_{1} L_{2} L_{3} C_{1} C_{2} C_{3} \omega^{6}
\end{aligned}
$$

from which the inductances and capacitances are determined according to three Larmor frequencies of interest.

To summarize, we have demonstrated the feasibility of double-resonance MACS experiments. MACS is an attractive option for performing microcoil-MAS experiments in the sense that a commercial CP-MAS probe can be used as it is without hardware modification, and the susceptibility-induced static-field inhomogeneity can effectively be suppressed. Double-resonance MACS can potentially be a versatile strategy for solid-state NMR analysis of intrinsically mass-limited samples when further progress is made in terms of rf-efficiency optimization, analysis and suppression of the sample heating effect due to the 
Eddy current induced by the spinning coil, and further increase in the spinning speed.

\section{Acknowledgment}

We thank Prof. K. Takegoshi at Kyoto University and Prof. M. Kitagawa at Osaka University for fruitful discussions on the subject. The A $\beta 42$ sample used in this work was provided by Prof. K. Irie and Dr. Y. Masuda. This work has been financially supported by the CREST program of Japan Science and Technology Agency.

\section{References}

[1] D.L. Olson, T.L. Peck, A.G. Webb, R.L. Magin and J.V. Sweedler, Science 270, 1967 (1995).

[2] K. Yamauchi, J.W.G. Janssen and A.P.M. Kentgens, J. Magn. Reson. 167, 87 (2004).

[3] A.P.M. Kentgens, J. Bart, P.J.M. van Bentum, A. Brinkmann, E.R.H. van Eck, J.G.E. Gardeniers, J.W.G. Janssen, P. Knijn, S. Vasa and M.H.W. Verkuijlen, J. Chem. Phys. 128, 052202 (2008).

[4] D.I. Hoult and R.E. Richards, J. Magn. Reson. 24, 71 (1976).

[5] A.G. Webb, S.C. Grant, J. Magn. Reson. B113, 83 (1996).

[6] E.R. Andrew, A. Bradbury and R.G. Eades, Nature 182, 1659 (1958).

[7] I.J. Lowe, Phys. Rev. Lett. 2, 285 (1959). 
[8] H. Janssen, A. Brinkmann, E.R.H. van Eck, J.M. van Bentum and A.P.M. Kentgens, J. Am. Chem. Soc. 128, 8722 (2006).

[9] K. Yamauchi and T. Asakura, Chem. Lett. 35, 426 (2006).

[10] M. Inukai and K. Takeda, Concepts in Magnetic Resonance 33B, 115 (2008).

[11] D. Sakellariou, G. le Goff and J.-F. Jacquinot, Nature 447, 694 (2007).

[12] A. Pines, M.G. Gibby and J.S. Waugh, J. Chem. Phys. 59, 569 (1973).

[13] K. Takeda, J. Magn. Reson. 192, 218 (2008).

[14] K. Takeda, Rev. Sci. Instrum. 78, 033103 (2007).

[15] P.M. Aguiar, J.-F. Jacquinot and D. Sakellariou, J. Magn. Reson. 200, 6 (2009).

[16] Y. Masuda, S. Uemura, A. Nakanishi, R. Ohashi, K. Takegoshi, T. Shimizu, T. Shirasawa, and K. Irie, Bioorg. Med. Chem. Lett. 18, 3206-3210 (2008).

[17] K. Takegoshi, S. Nakamura and T. Terao, Chem. Phys. Lett. 344, 631 (2001).

[18] K. Takegoshi, S. Nakamura and T. Terao, J. Chem. Phys. 118, 2325 (2003). 


\section{Figure Captions}

\section{Figure 1}

(a) A circuit diagram for implementing double-resonance MACS experiments. The two inductors $L_{1}$ and $L_{2}$ were wound with $80 \mu \mathrm{m}$ polyurethane-coated copper wire into $0.5 \mathrm{~mm} \phi$ microcoils, and a doubly-tuned MACS resonator was fabricated with two $1 \times 0.5 \times 0.5 \mathrm{~mm}$ chip capacitors, as shown in (b). (c) illustrates how the coils and the capacitors are supported by a Shapal-M holder.

\section{Figure 2}

The contour plots in (a) and (b) describe the $L_{1}$ and $L_{2}$ values required to tune the circuit at both $300 \mathrm{MHz}$ and $75 \mathrm{MHz}$ for a given set of the capacitances $C_{1}$ and $C_{2}$. These plots are calculated from Eqs. (2)-(5), and in the present work, $L_{1}=11 \mathrm{nH}, L_{2}=43 \mathrm{nH}, C_{1}=33 \mathrm{pF}$, and $C_{2}=82 \mathrm{pF}$.

\section{Figure 3}

(a) Radiofrequency-power dependence of the nutation frequencies for the ${ }^{13} \mathrm{C}$ and ${ }^{1} \mathrm{H}$ spins in a Varian $5 \mathrm{~mm}$ CP-MAS probe at $75 \mathrm{MHz}$ and $300 \mathrm{MHz}$. The magnetic field was $7 \mathrm{~T}$. The blue symbols represent the ${ }^{13} \mathrm{C}$ nutation frequencies with (filled squares) and without (open squares) the doubly-tuned MACS resonator, and the red symbols represent the ${ }^{1} \mathrm{H}$ nutation frequencies with (filled circles) and without (open circles) the MACS resonator. The data points were fitted by a square root function of the form $y=a \sqrt{x}$, and the coefficient $a$ is depicted for each set of the data. (b) A ${ }^{13} \mathrm{C}$ CP-MAS spectrum of polycrystalline ${ }^{13} \mathrm{C}-{ }^{15} \mathrm{~N}$-labeled L-alanine obtained without the doubly-tuned MACS resonator. The sample $(\sim 0.1 \mathrm{mg})$ was packed in a capillary tube and 
put into the rotor using a spacer. (c) $\mathrm{A}{ }^{13} \mathrm{C}$ spectrum of the identical sample obtained by employing the doubly-tuned MACS resonator. The numbers 7.0, 7.3, and 7.3 indicate the enhancement factors of the ${ }^{13} \mathrm{C}$ peak intensities for the methyl, methine, and carboxyl carbon sites in L-alanine, while the numbers in the parentheses are the overall enhancement factors in the signal-to-noise ratio by taking account of the additional noise arising from the MACS insert. In both (b) and (c), the spinning frequency was $8 \mathrm{kHz}$, and the signals were accumulated over 1,000 times under TPPM ${ }^{1} \mathrm{H}$ decoupling with an intensity of $100 \mathrm{kHz}$.

\section{Figure 4}

(a) ${ }^{13} \mathrm{C}$ CP-MAS spectrum of $\mathrm{A} \beta 42\left(\sim 0.1 \mathrm{mg}\right.$ of sample) partially ${ }^{13} \mathrm{C}$ labeled at the 35-Met and 42-Ala amino acid residues. The sample was synthesized as reported previously[16]. The ${ }^{13} \mathrm{C}$ NMR signals were accumulated over 180,000 times with a recycle delay of $1 \mathrm{~s}$. The spinning frequency was 10 $\mathrm{kHz}$. (b) A two-dimensional ${ }^{13} \mathrm{C}-{ }^{13} \mathrm{C}$ DARR correlation spectrum in uniformly ${ }^{13}$ C-labelled polycrystalline histidine ( $\sim 0.1 \mathrm{mg}$ of sample). 128 hypercomplex data points were acquired in the indirect dimension, and each of them was accumulated over 1280 times with a recycle delay of $0.5 \mathrm{~s}$. The spinning frequency was $5 \mathrm{kHz}$. Both data were acquired using the doubly-tuned MACS resonator.

\section{Figure 5}

Comparison of the resonance line width of the methyl ${ }^{13} \mathrm{C}$ in a polycrystalline sample of ${ }^{13} \mathrm{C}-{ }^{15} \mathrm{~N}$-labeled L-alanine for the MACS and piggy-back schemes under TPPM ${ }^{1} \mathrm{H}$ decoupling with an intensity of $100 \mathrm{kHz}$. The spinning frequency was $8 \mathrm{kHz}$. 


\section{Figure 6}

A circuit diagram for a triply-tuned MACS resonator. $V_{1}, V_{2}$, and $V_{3}$ represent electromotive forces induced by the primary circuit in the probe. 


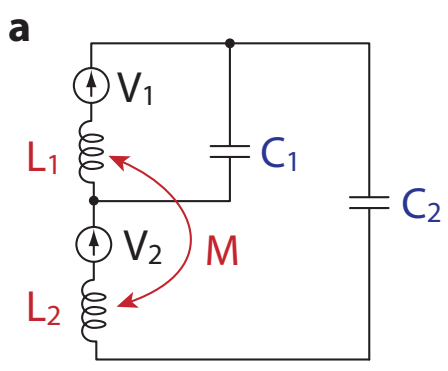

b

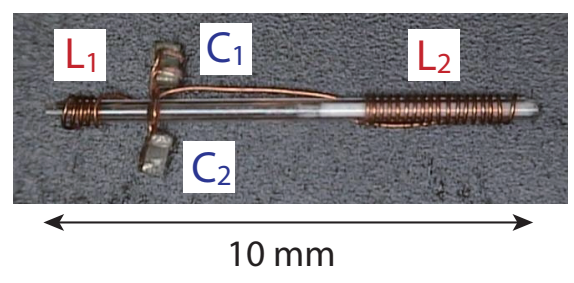

C

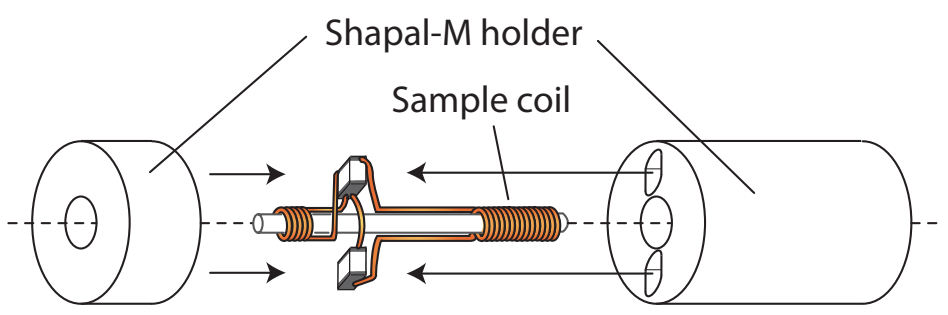

Fig. 1

Doubre-resonance...

Inukai et al. 

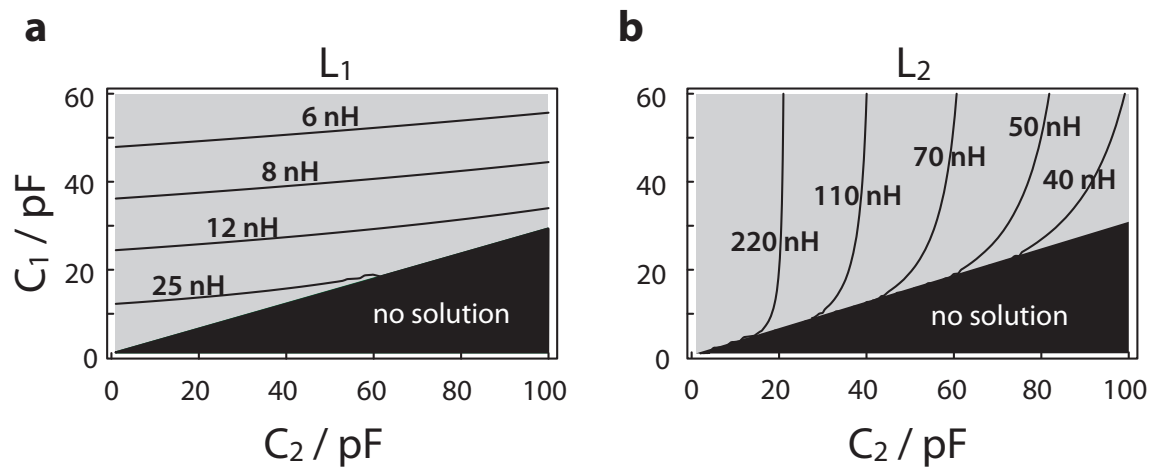

Fig. 2

Doubre-resonance...

Inukai et al. 
a

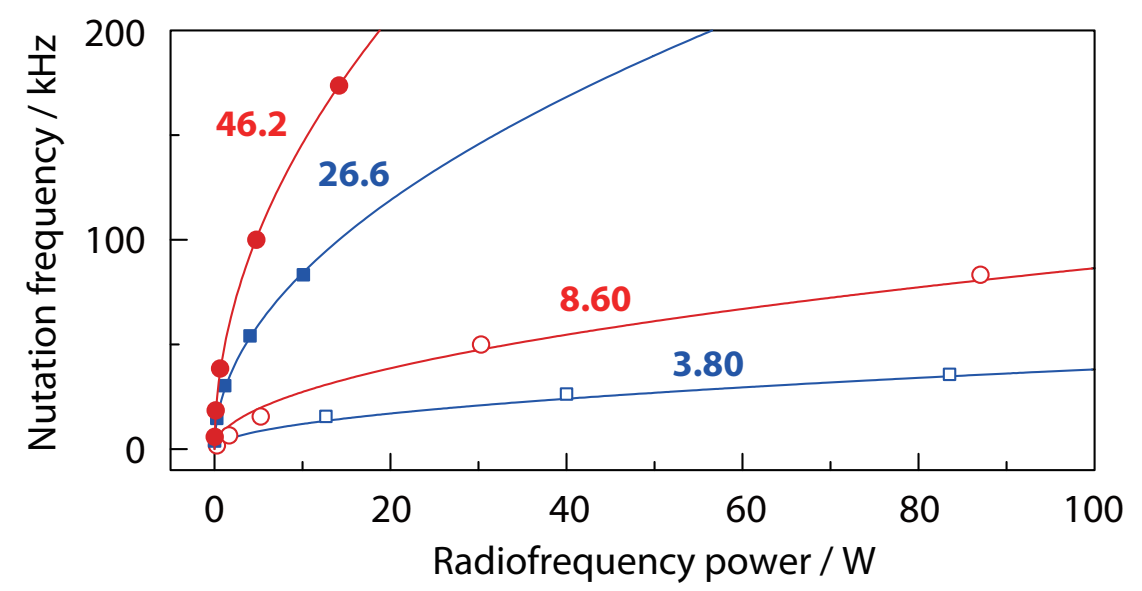

b

C

7.0

(6.3)

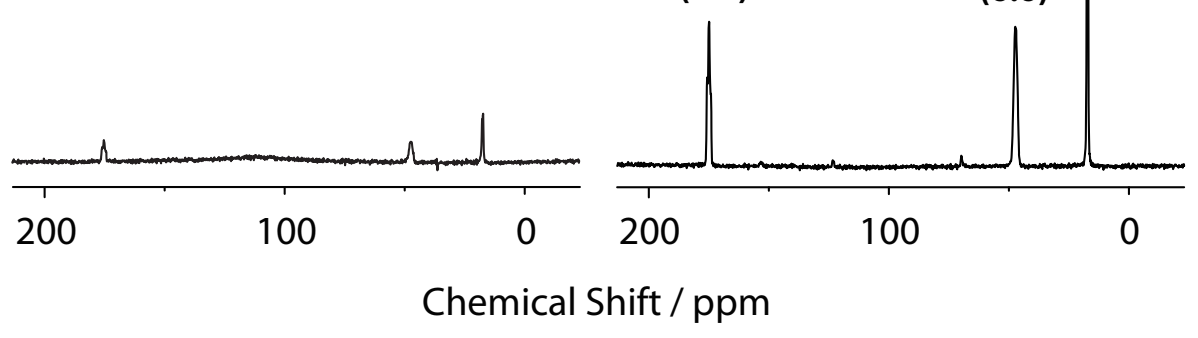

7.3

(6.3)

7.3

(6.6)

Fig. 3

Doubre-resonance...

Inukai et al. 
a

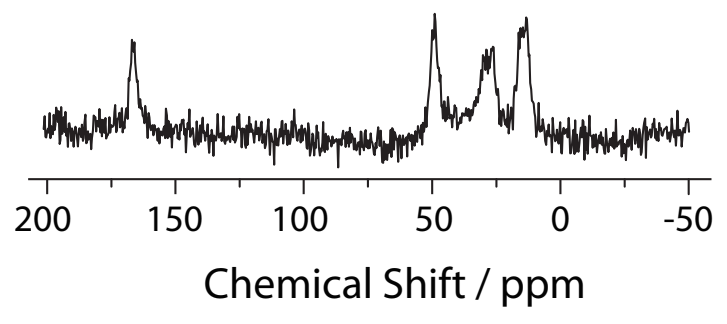

b

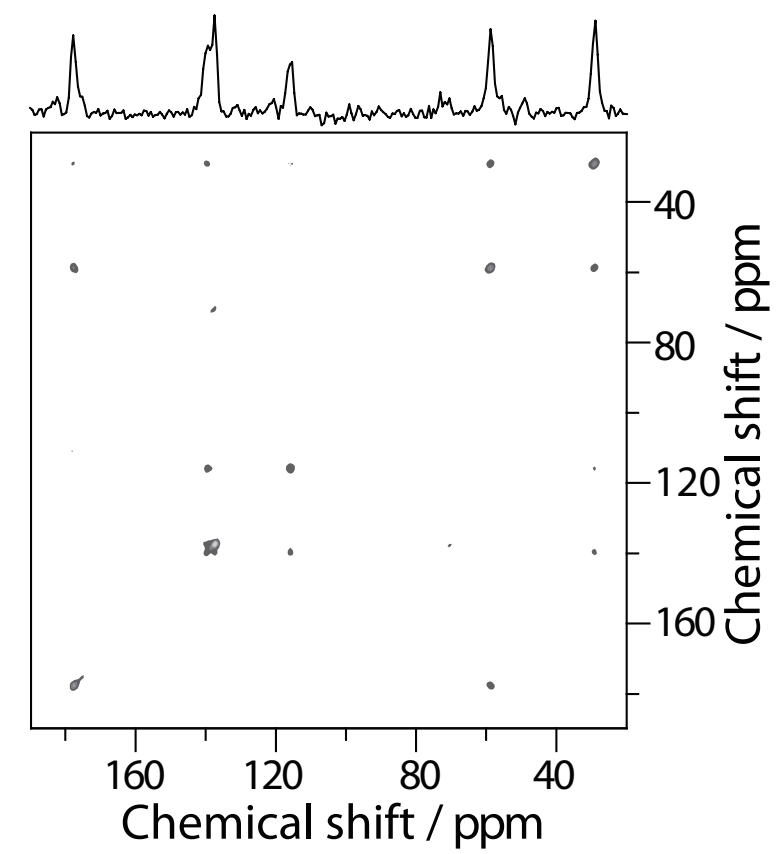

Fig. 4

Doubre-resonance...

Inukai et al. 


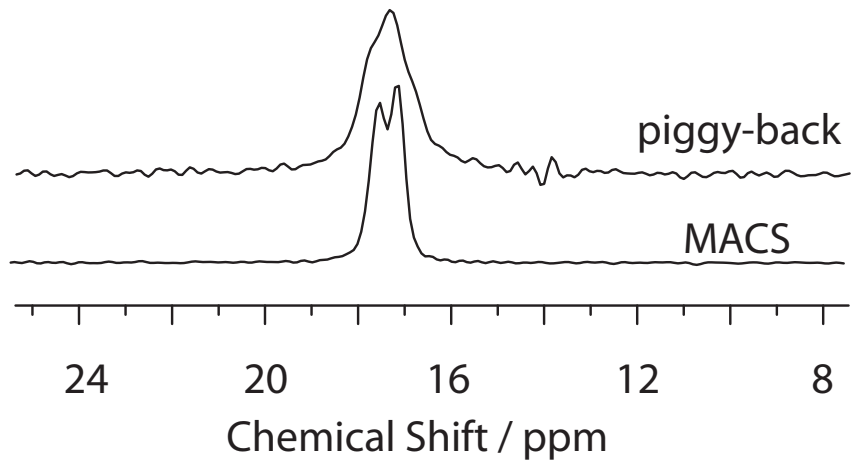

Fig. 5

Doubre-resonance...

Inukai et al. 


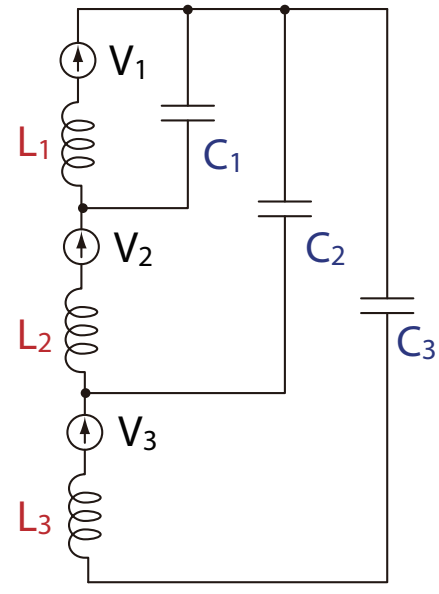

Fig. 6

Doubre-resonance...

Inukai et al. 